\title{
Design and Development of K-Band FMCW Radar for Nano-Drone Detection
}

\author{
Safiah Zulkifli and Alessio Balleri \\ Centre for Electronic Warfare, Information and Cyber, Cranfield University, Defence Academy of the UK, Shrivenham, SN6 8LA \\ \{Safiah.Binti-zulkifli, a.balleri\}@cranfield.ac.uk
}

\begin{abstract}
Nano-drones, are insect-like size drones with a capability of intrusion to provide intelligence and potentially violate secure establishments and public privacy rights. Nanodrones are already an existing technology which is becoming more and more available, portable, affordable and easily operated. As such, they may soon become a plausible defence and security threat. This paper presents the design and development of a K-Band FMCW radar prototype for nanodrone detection. The FMCW radar prototype consists of connectorised components operating at a carrier frequency of $24 \mathrm{GHz}$ and offer high parameter selection flexibility. Experiments have been carried out in order to evaluate the system performance. Results show that a small Arcade PICO Drone Nano Quadcopter (smaller than $5 \mathrm{~cm}$ ) could be detected, and that its micro-Doppler signature could be extracted from data.
\end{abstract}

Keywords-Home-Brew Radar, Micro-Doppler Extraction, Micro-UAV, Nano-Drones, K-Band FMCW Prototype

\section{INTRODUCTION}

The greatest technological transformation towards unmanned aerial vehicle started with the transition from fullsize aircraft to large remotely controlled and unpiloted aerial platforms. Then slowly the size of drones has significantly reduced to provide unmanned solutions that have become more portable, economical and affordable. Drones are no longer restricted to the military, and they have become a technology readily available for civilians, either for industrial or private purposes. A recent study has claimed that advanced research in nano-drones, e.g. smaller versions of drones up to insect-size, might result in a much more vulnerable environment to the society as drones may soon become a perfect tool for stealthy surveillance [1]. Smaller drones are characterised by a low Radar Cross Section (RCS) and, as a result, detecting them with conventional radar becomes a challenging task. Increasing the radar operating frequency (i.e. shorter wavelength) is a possible way to increase the target RCS. However, at the same time this reduces the radar maximum detection range as attenuation due to atmosphere and dissipation losses grow with frequency. As the radar performance is usually evaluated by the ability to detect a specific target at a given range, it is important to compromise between the operating frequency and the detectable range.
One of the techniques that have been deployed in microUAV detection is the extraction of micro-Doppler signature. Micro-Doppler signatures are Doppler signatures induced by micro-motions of the target, like the rotation of the propeller blades. Since micro-UAVs can hover or move at a relatively low speed, they are often characterised by a small main body Doppler return. However, small rotating or vibrating parts of micro-UAVs can largely contribute to the micro-Doppler.

A large and growing body of literature has investigated UAV detection and classification by micro-Doppler techniques. In particular, previous studies have focused on mini-UAVs (MUAVs), with typical dimensions in the range between $30 \mathrm{~cm}$ to less than $2 \mathrm{~m}$ and micro-UAVs (MAVs) with less than $30 \mathrm{~cm}$ wingspans [2]. Although there are no standard global dimension of nano-UAVs (NAVs) yet, a program that has been launched to design the NAV have specified the size to be less than $10 \mathrm{~cm}$ wingspan [3]. Authors in [4] made one the first attempts in mini-UAVs classification, by using an $\mathrm{X}$-band $\mathrm{CW}$ radar to investigate the signatures of a variety of target types including small fixed-wing planes, small helicopters (main rotor size between $71 \mathrm{~cm}-104 \mathrm{~cm}$ ) and a quadcopter (blade size $20 \mathrm{~cm}$ ). In [5], the characteristic features extracted from the signatures of the mini-UAVs measured in [4] were used to investigate how to distinguish drones from birds. A different radar setup was deployed for micro-UAV detection in [6] and [7], whereby the authors developed an S-band $(2.4 \mathrm{GHz})$ multistatic pulsed radar to detect a drone (DJI Phantom Vision 2+) versus birds and to differentiate between loaded and unloaded drones. In [8] and [9], FMCW radars were used to detect the Hexa-copter BumbleBee F820, with a propeller diameter of $38 \mathrm{~cm}$, at Kband and to detect a DJI Phantom Drone Quadcopter at Wband. It was demonstrated that the proposed FMCW radar could provide an extra advantage with respect to detection range due to a frequency modification that enabled measurements of time delays and in return provide range information. Similar research has been carried out in [10] [13] to investigate the micro-Doppler signatures of different types of drones (from MUAVs to MAVs) and how these could be used to classify the targets accordingly. Together, all these studies provide an important insight into micro-Doppler techniques applied to detection and classification of drones. Despite the work described above, the existing literature lacks 
of detailed investigations focussing on detection of nanodrones (or the insect-size drone). To the best of our knowledge, the smallest blade sizes been studied in the current literature are $13 \mathrm{~cm}$ and $13.5 \mathrm{~cm}$, for blades belonging to DJI Phantom 3 Standard and DJI Mavic Air drones, respectively. Also, the total body size of both these drones is much bigger than the size of their blades. Because there is a large growing interest in pocket-size drone mass production, there is a need to give an account of nano-target detection as well [14].

This paper investigates the possibility of nano-drone detection using a home-brew radar. The radar system is designed and developed, using connectorised off-the-shelf components at $24 \mathrm{GHz}$. Unlike analogous off-the-shelf integrated radars, the developed K-band FMCW radar prototype offers a high level of parameters selection flexibility and as such allowed us to change key radar waveform design characteristics to analyse and obtain the desired performance. Several experiments have been carried out on an Arcade PICO drone to extract its micro-Doppler signature.

\section{RADAR DESIGN MODEL}

A periodic signal is continuously transmitted at a carrier frequency $f_{o}$ that consists of a sequence of linear frequency modulated ramps

$$
S_{T X}(t)=A_{T X} \cos \left[2 \pi f_{o} t+\left(\frac{B}{T_{R}}\right) \pi t^{2}\right]
$$

of amplitude $A_{T X}$, bandwidth $B$ and ramp duration $T_{R}$. The transmit signal propagates to the target which produces an echo signal

$$
\begin{aligned}
& S_{R X}(t)=A_{R X} \cos [ 2 \pi f_{o}\left(t-t_{0}\right) \\
&\left.+\left(\frac{B}{T_{R}}\right) \pi\left(t-t_{0}\right)^{2}\right]
\end{aligned}
$$

where $A_{R X}$ is the amplitude of the received signal and $t_{o}$ is the time delay that accounts for the propagation path. FMCW radars that employ a double balanced mixer on receive generate an output signal

$$
S_{f_{b}}(t)=A_{f_{b}} \cos \left(2 \pi f_{0} t_{0}+2 \pi \gamma t_{o} t-\pi \gamma t_{0}{ }^{2}\right)
$$

of amplitude $A_{f_{b}}$ which is obtained by mixing the received signal $S_{R X}(t)$ with a copy of the transmitted signal $S_{T X}(t)$. The frequency of the signal $S_{f_{b}}(t)$ provides the target range information via a calculation of the time delay $t_{o}$. The primary parameter in this design is the slope of the ramp $\gamma=B / T_{R}$ which, for each range bin, determines the expected beat frequency

$$
f_{b}=\frac{B}{T_{R}} \cdot \frac{2 R_{0}}{c}
$$

If an object is moving at a relative radial velocity $v$ with respect to the radar, the time delay measured by the $n$-th ramp is $t_{0}=2\left(R_{0}+v n T_{R}\right) / c$ and the target Doppler shift induced by the movement of the target can be extracted by comparing the phase of the Fourier transform of the beat signal from ramp to ramp.

Our underlying strategy to this design was to minimise the $\mathrm{RF}$ components and the cost of the radar prototype. To achieve this, we excluded the use of IQ demodulator components that would have represented an additional cost to the prototype. For this radar design, a target at a range $R_{0}$ produces a beat signal with a spectrum characterised by a positive and a negative contribution centred at the target range, as shown in Fig 1. The use of IQ demodulators, instead, provides a onesided spectrum and therefore no blind range limitations. Selecting a high frequency bandwidth results in a much wider separation of the two spectrum components as it shifts the resulting beat frequency away from DC and the overlapping zone. This limitation does not affect targets that are farther away from the radar as these generate non-overlapping spectrum components. The minimum requirement in this case is that the range of the target must be higher than the range resolution of the radar. One must also bear in mind that, the greater the distance of the target, the higher the transmitted power required to optimise target detection.

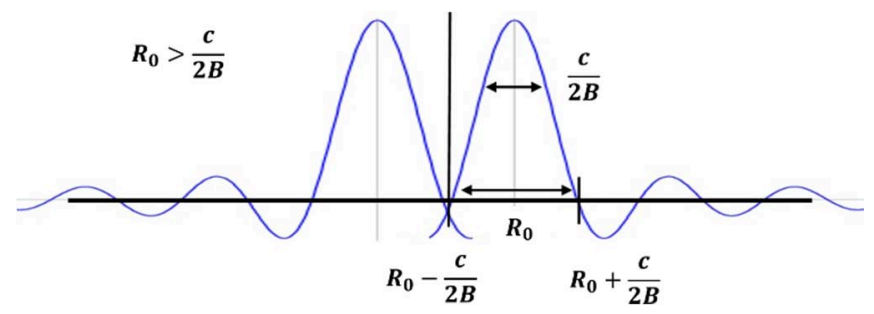

Fig 1: Two-Sided Spectrum

Doppler signatures are extracted by a double FFT and a time-frequency analysis. A fast-time FFT is applied ramp to ramp to construct a 2D matrix known as Range-Time Intensity (RTI). Then, a Range-Doppler map (RDM) is structured by applying second FFT for each range bin of the RTI matrix which will then transform the previous slow-time axis into the Doppler information. Lastly, a Short-Time Fourier Transform (STFT) is applied at the selected target range bin from the RDM to extract the micro-Doppler signature from the RTI.

\section{PROTOTYPE IMPLEMENTATION}

The K-band FMCW radar transmits linear frequency modulated ramps of a user-selectable duration spanning the frequency bandwidth from $24.58 \mathrm{GHz}$ to $25.06 \mathrm{GHz}$ [15]. A linear ramp voltage signal is generated by a Keysight Waveform Generator 33600A (see Fig 2) and fed into a HMC739LP4 Voltage Controlled Oscillator (VCO) to provide the frequency modulated signal at K-band. The VCO is an 
evaluation board manufactured by Hittite with an integrated chip mounted on the board.

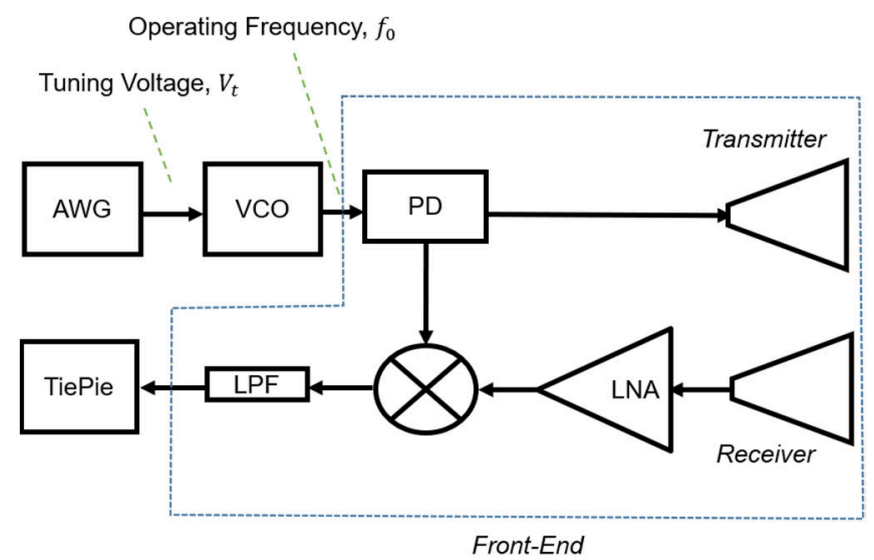

Fig 2: Block Diagram of K-Band Radar Prototype

The output of the VCO is then passed to a mini-circuits ZC2PD-18263-S+ power divider which splits it into two signals; one is directed to the transmit antenna and the other one is fed to the LO port of a Marki Microwave MM1-1140HS double-balanced mixer and is used as the beating copy of the transmitted signal. Target echoes are collected by the receive antenna and are passed to a HD30172 Low Noise Amplifier (LNA) for signal amplification. The amplified received signal is then flowed to the RF port of the mixer and mixed down with the copy of the transmitted signal available at the LO port of the mixer. The IF product is filtered with a mini-circuits VLF-45+ low pass filter before being digitised with a Handyscope HS5 USB oscilloscope. The RF components of the radar is illustrated in Fig 3. The transmit and receive antennas are identical with a gain of $18 \mathrm{dBi}$, length $135 \mathrm{~mm}$ and aperture $40 \times 50 \mathrm{~mm}$. During the experiments both antennas were mounted on a bracket $1.5 \mathrm{~m}$ above the floor.

The main advantage of this prototype is that it provides full control of the radar components and the flexibility to choose the radar parameters, and hence the radar performance, on a case to case as desired. The operational frequency range of the $\mathrm{VCO}$, for example, is between $23.8 \mathrm{GHz}$ to $26.8 \mathrm{GHz}$ allowing a wide selection of the frequency span and bandwidth within the system linear response interval.

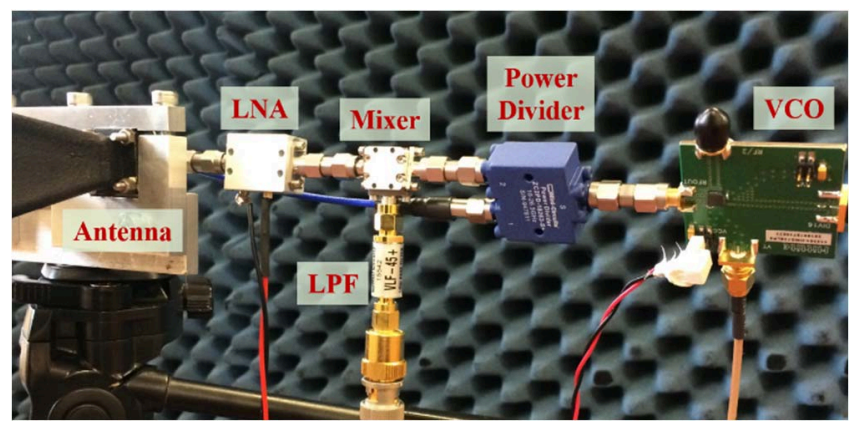

Fig 3: A Close-Up of Radar Prototype

\section{PERformance EVAluation}

The experiments were carried out in the Radar Laboratory of Cranfield University, at the Defence Academy of the UK in Shrivenham, to evaluate the capability of the radar prototype. The target used in these experiments was an Arcade PICO Drone Nano Quadcopter, with a body size of approximately 3 $\mathrm{cm}$ and consisting of 4 propellers carrying $1.2 \mathrm{~cm}$ long blades, as illustrated in Fig 4.

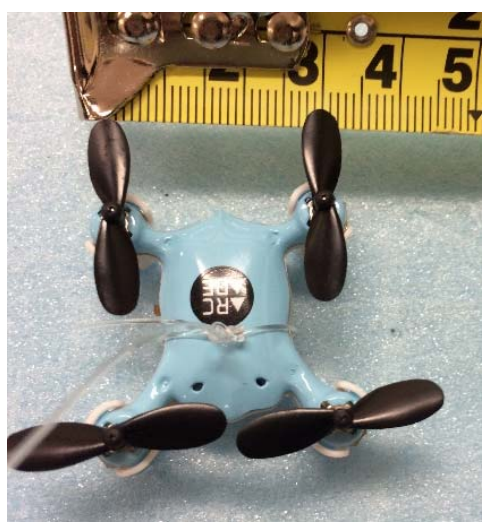

Fig 4: Close-Up of the Target: ARCADE Pico Drone

During the measurements, the drone was tied up to a thin fisher line and hanged to the ceilings from the top and from the bottom to the floor, to help the drone hover in one place and to minimise uncontrollable trajectories. The drone was placed about antenna boresight and approximately $1.5 \mathrm{~m}$ above the ground (at the same height as the antennas of the radar prototype). A Radar Absorbent Material (RAM) panel was placed behind the radar prototype and another RAM panel was placed behind the target to limit multipath effects. The target range from the radar was about $3 \mathrm{~m}$. Fig 5 shows a photo of the hanging drone which was then switched on for data collection with the rotating blades.

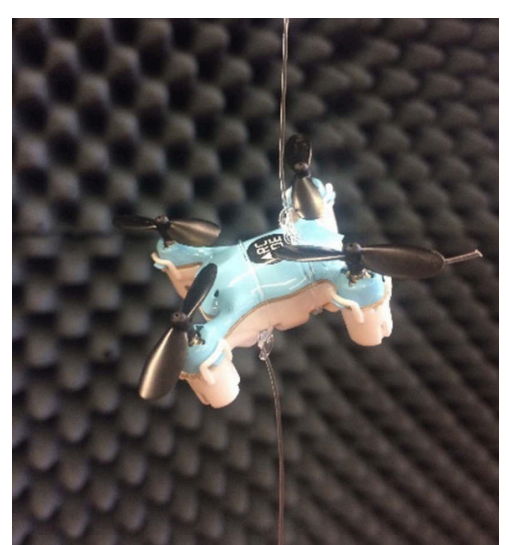

Fig 5: Hanging Drone for Data Collection

The data was sampled with a $500 \mathrm{kHz}$ sampling frequency and results generated for integration times of $2 \mathrm{~s}$. A first set of measurements were collected with a ramp duration of $1 \mathrm{~ms}$ (Case I). Fig 6 shows the range doppler map of the target obtained while the blades were continuously rotating. Because of the presence of the fishing line, and due to the 
spinning blades, during the measurements the body of the drone moved on a small circular trajectory within the fixed place. The drone is detected at approximately $2.42 \mathrm{~m}$ away from the radar. The highest peak signal return corresponds to the main body of the drone which because of the rotational trajectory, was slightly oscillating about zero-Doppler. The exact rotational rate of the blades is unknown, but the rangeDoppler map shows maximum Doppler frequency returns around $400 \mathrm{~Hz}$.

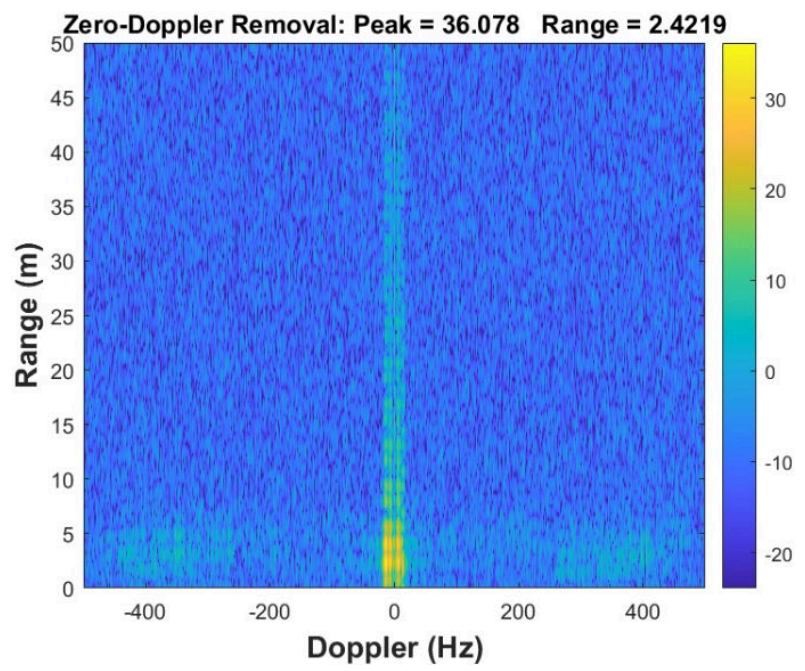

Fig 6: Range-Doppler Map of the Rotating Pico-Drone within $2 \mathrm{~s}$

Fig 7 shows the STFT for the target range bin. The Helicopter Rotor Modulation (HERM) lines induced by the propeller blades of the drone are clearly visible. There are 3 lines at positive and negative Doppler frequencies which result from the complex interaction between all the target components. From these measurements alone, determining the rotational rate of the propeller blade is not obvious but the observed Doppler returns suggest periodicities of about 25,000 RPM. The length of STFT window was $60 \mathrm{~ms}$. Results demonstrate that even smaller size drones, built-in plastic material body can be detectable in an enclosed building.

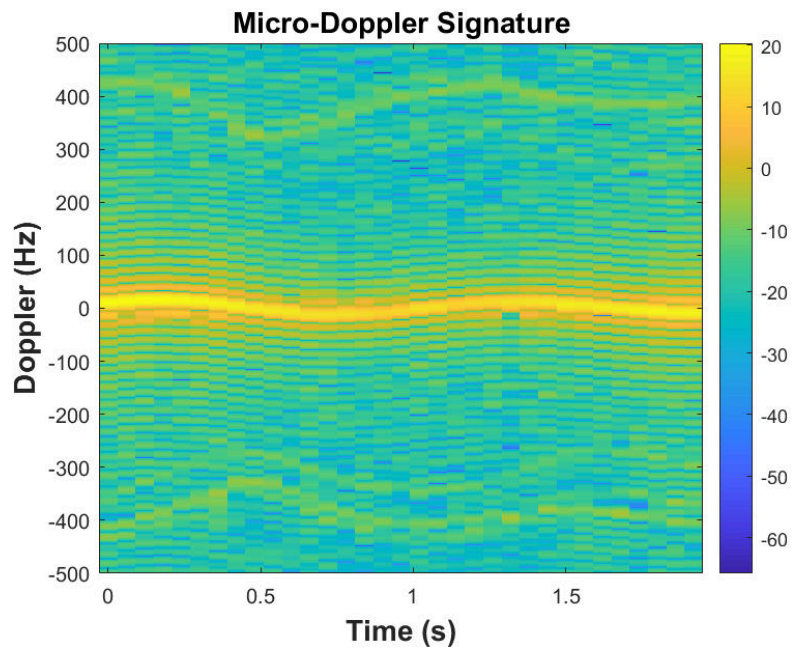

Fig 7: Micro-Doppler Signature of Pico Drone in $2 \mathrm{~s}$
Data of the same target was also collected for a shorter ramp duration of $0.5 \mathrm{~ms}$ (Case II). The data was sampled at $500 \mathrm{kHz}$ and results obtained with the same integration time of $2 \mathrm{~s}$. The drone was detected $2.5 \mathrm{~m}$ away from the radar. Fig 8 shows the target Doppler returns over a wider Doppler bandwidth and, for this case, the maximum Doppler frequency return is between $500 \mathrm{~Hz}$ to $600 \mathrm{~Hz}$.

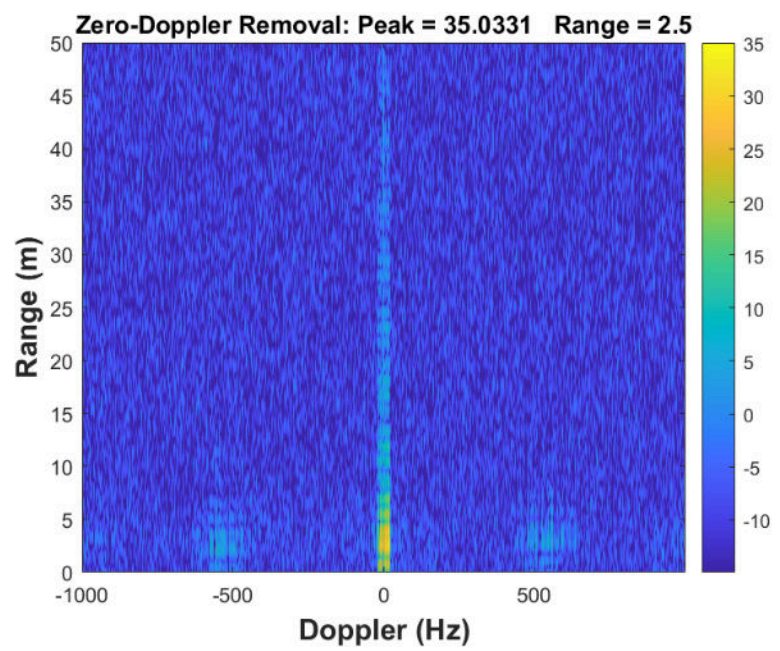

Fig 8: Range-Doppler Map of the Rotating Pico-Drone within $2 \mathrm{~s}$

The length of STFT window used to extract microDoppler signature was $60 \mathrm{~ms}$. Fig 9 shows the target microDoppler signature with the same Doppler resolution over a larger Doppler bandwidth. The blade behaviour is more visible compared to Fig 7. Again, the plot corroborates that, for this case, the maximum Doppler frequency returns were contributing approximately around $500 \mathrm{~Hz}$. From the two cases discussed, it is evident that there is a trade-off in having a shorter ramp duration and a longer ramp duration. One of the key requirements is for the ramp duration $T_{R}$ to be at least twice the maximum Doppler return of the drone to avoid ambiguous Doppler measurement.

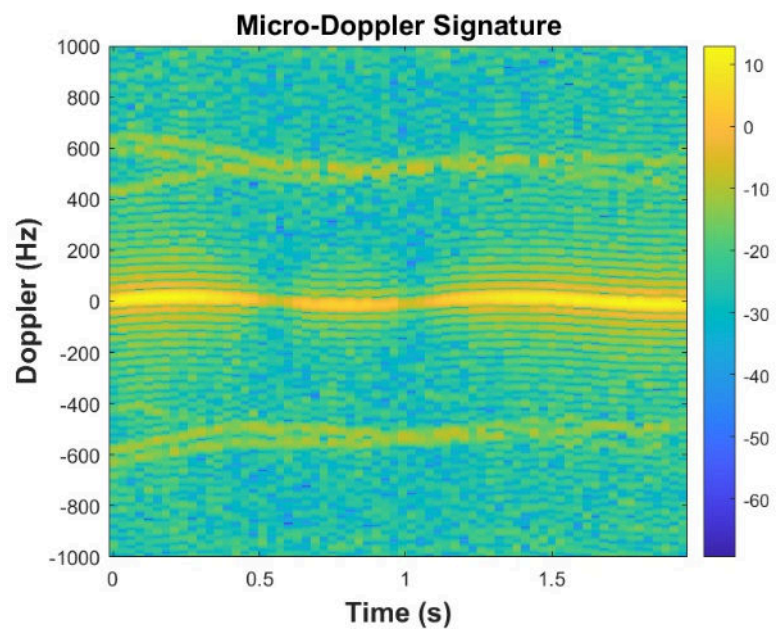

Fig 9: Micro-Doppler Signature of Pico Drone in $2 \mathrm{~s}$ 


\section{CONCLUSION}

This project was undertaken to design, develop and prototyping a low-cost $\mathrm{K}$-band $(24 \mathrm{GHz}) \mathrm{FMCW}$ radar using the off-the-shelf connectorised components. The radar design offers a high parameter selection flexibility at a frequency range of $24 \mathrm{GHz}$. Results have demonstrated the workability of the radar by showing that the nano-drone could be detected and its micro-Doppler signature extracted using a STFT.

\section{ACKNOWLEDGMENT}

The authors would like to thank the School of Aerospace Engineering, Universiti Sains Malaysia, Malaysia and the Malaysian Ministry of Higher Education for sponsoring the $\mathrm{PhD}$ studies of Safiah Zulkifli at Cranfield University.

\section{REFERENCES}

[1] 'The Drone Threat to National Security'. Available at https://www.scientificamerican.com/article/the-drone-threat-tonational-security/, accessed in March 2020

[2] Miller, P. M. and Goodrich, M. K., Mini, Micro and Swarming Unmanned Aerial Vehicles: A Baseline Study. Library of Congress. Nov 2006

[3] T. Hylton, C. Martin, R. Tun, and V. Castelli, "The DARPA nano air vehicle program," 50 ${ }^{\text {th }}$ AIAA Aerospace Sciences Meeting including the New Horizons Forum and Aerospace Exposition, Tennessee, 2012

[4] P. Molchanov, K. Egiazarian, J. Astola, R. I. A. Harmanny and J. J. M. de Wit, "Classification of small UAVs and birds by micro-Doppler signatures," 2013 European Radar Conference, Nuremberg, 2013, pp. 172-175.
[5] J. J. M. de Wit, R. I. A. Harmanny and P. Molchanov, "Radar microDoppler feature extraction using the Singular Value Decomposition," 2014 International Radar Conference, Lille, 2014, pp. 1-6.

[6] M. Ritchie, F. Fioranelli, H. Griffiths and B. Torvik, "Monostatic and bistatic radar measurements of birds and micro-drone," 2016 IEEE Radar Conference (RadarConf), Philadelphia, PA, 2016, pp. 1-5.

[7] M. Ritchie, F. Fioranelli, H. Borrion and H. Griffiths, "Multistatic micro-Doppler radar feature extraction for classification of unloaded/loaded micro-drones," in IET Radar, Sonar \& Navigation, vol. 11, no. 1, pp. 116-124, 12017.

[8] D. Shin, D. Jung, D. Kim, J. Ham and S. Park, "A Distributed FMCW Radar System Based on Fiber-Optic Links for Small Drone Detection," in IEEE Transactions on Instrumentation and Measurement, vol. 66, no. 2, pp. 340-347, Feb. 2017

[9] M. Caris, W. Johannes, S. Sieger, V. Port and S. Stanko, "Detection of small UAS with W-band radar," 2017 18th International Radar Symposium (IRS), Prague, 2017, pp. 1-6

[10] L. Fuhrmann, O. Biallawons, J. Klare, R. Panhuber, R. Klenke and J. Ender, "Micro-Doppler analysis and classification of UAVs at $\mathrm{Ka}$ band," 2017 18th International Radar Symposium (IRS), Prague, 2017, pp. 1-9

[11] S. Björklund, "Target Detection and Classification of Small Drones by Boosting on Radar Micro-Doppler," 2018 15th European Radar Conference (EuRAD), Madrid, 2018, pp. 182-185.

[12] S. Rahman., D.A. Robertson, "Radar micro-Doppler signatures of drones and birds at K-band and W-band," Sci Rep 8, 17396, 2018

[13] A. Aldowesh, T. BinKhamis, T. Alnuaim and A. Alzogaiby, "Low Power Digital Array Radar for Drone Detection and Micro-Doppler Classification," 2019 Signal Processing Symposium (SPSympo), Krakow, Poland, 2019, pp. 203-206

[14] S. Zulkifli and A. Balleri, "FMCW Radar Prototype Development for Detection and Classification of Nano-Targets," in 2020 IEEE International Radar Conference, April 2020

[15] 'UK Frequency Allocation Table (UKFAT)'. Available at http://static.ofcom.org.uk/static/spectrum/fat.html. Accessed in 16July-2020 